\title{
A Review Paper on General Concepts of "Artificial Intelligence and Machine Learning"
}

\author{
Mahind Rupali ${ }^{1}$, Patil Amit ${ }^{2}$ \\ Assistant Professor, Computer Science \& Engg, DACOE, Karad, India ${ }^{1}$ \\ Student, Computer Science \& Engg, DACOE, Karad, India ${ }^{2}$
}

\begin{abstract}
Long days ago, there was all kind of work which is only done by the humans. There were no such machines and technologies like today. At that time, science is not developed and technologies were not invented. So the working is totally dependent on the peoples and humans have recognised that "Today's science is the tomorrow's technology". New superiorly advanced technologies are not less than blessing of god. Adaptive inventions for reducing the human work and bright future were invented which is simply called as Artificial Intelligence and Machine learning. Even though there were many false assumptions at the early beginning, we are witnessing a new era of errorless technology and superior science. This review involves the general concepts of artificial intelligence and machine learning.
\end{abstract}

Keywords: Digitalization, reasoning, general AI, supervised learning, unsupervised learning, humanoids.

\section{INTRODUCTION}

As the humans have proved their presence on the earth, it implemented the basic perspectives of artificial is important that every individual should understand what intelligence. They concluded that the artificial intelligence artificial intelligence and machine learning is going to is a combination of reasoning, learning, perception, mean for the human race .As a nice poem summarized by linguistic approach and problem solving.

W. H. Auden which gives the relationship between human Niklas Lavesson[3] also have described about the life and robotics:

Those who will not reason

Perish in the act;

Those who will not act

Perish for that reason.

The poem explains that "Fittest survival" i.e. only those humans/machines will survive who prove their existence by their best performance, high intelligence and maximum capacity. Hence it's high time and precipice of one of the most magnificent discoveries of supremacy since man learnt to create tools and fire. It is a road that once we walk ahead; there will be no turning back, once we achieve machine super intelligence which will be selflearning, completely automatic and self-improving.

As we see any sci-fi movie like iron man, star wars, terminator etc. Which vary in completely equipped super hero's to the world destroying super robots .Even if anyone has seen missile or space shuttle launching, automatically driven cars, or simple robots which help in household chores to hard power full muscle works, which are very great examples of human intelligence. No doubt that artificial intelligence and machine learning are the two hottest buzzword all over the world right now, and often seem to be used interchangeably.

\section{RELATED WORK}

Peter Norvig and Stuart Russell [1] in their research paper "Artificial intelligence: A modern approach" have supervised type of machine learning .The ambition of this review is to introduce the types of machine learning such as supervised, unsupervised and reinforcement etc. The review also explores the applications of AI and machine learning in real time.

George F Ludger [10] who described structures and strategies for artificial intelligence. The review also contains the methodologies of artificial intelligence such as weak artificial intelligence and strong artificial intelligence. It also considers the current real world applications and current processes in artificial intelligence.

\section{III .ARTIFICIAL INTELLIGANCE (AI)}

According to father of artificial intelligence John McCarthy, who coined the term "Artificial intelligence" in 1956, said that "It is the combination of science and engineering to make intelligent devices for human welfare."

"Artificial intelligence is an intellect [13] that is much smarter than the best human brain in practically every field, including computer science and linguistic logic."It is a modern method of machines which will do muscle work and illustrate complex questions in a "intellectual" manner. It is concerned with the basic and most important aspects in our life i.e. philosophy, computer science, mathematics, linguistics, biology, neuron science, sociology etc. AI plays a very important role to exhibit intelligent behaviour, to learn, demonstrate and give advice to the user. 
IARJSET

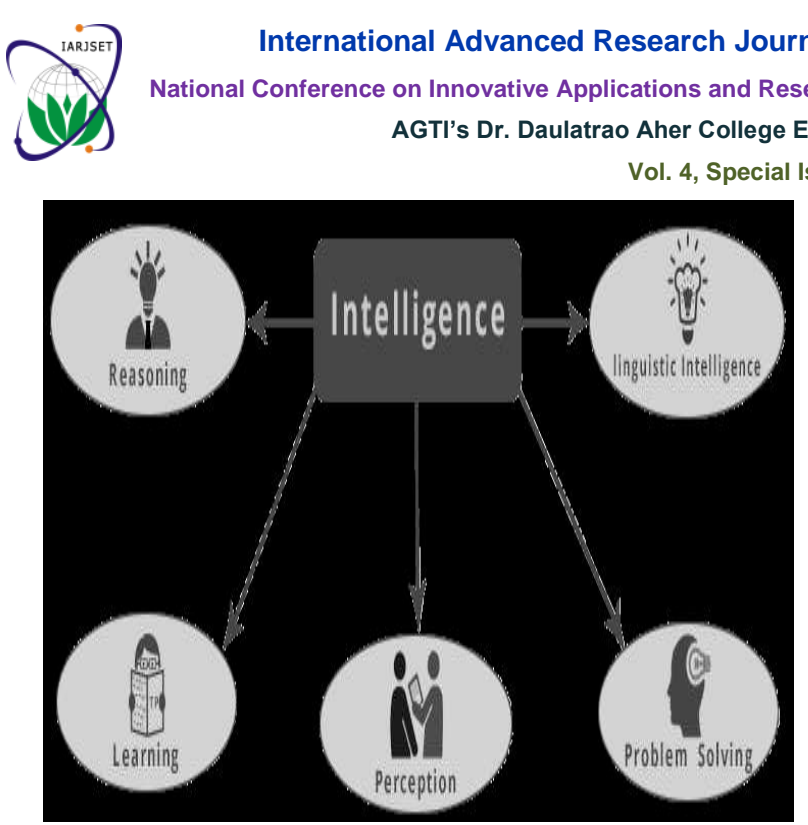

Fig. III. a Diagram of sectors in AI

Artificial General Intelligence or AGI is a system that defines that the machine can do intellectual behaviour as the humans can do many processes at a time. A broader view to artificial intelligence is that it can be the combination of learning, perception, problem solving and adapting new solutions to the system. It also involves linguistic logic and reasoning [1].

Artificial intelligence has 2 types:

1. Weak AI.

2. Strong AI.

\section{Weak AI.}

The principle of Weak AI is that the machines behave as if they are intelligent. Weak AI proves that virtual abilities like thinking, talking, moving can be done by machine if they are programmed in that manner. E.g. In the chess game, the computer can play and move players automatically. The computer does not have thinking ability but in actual it is programmed so that the computer always takes right step.

\section{Strong AI:}

The principle of Strong AI is that the machines will do calculations and think itself and will predict the answer in future. E.g. The artificial intellectual supercomputer 'WATSON' invented by IBM. Thus in future, there will be definitely such machines or may be humanoids which will do its own work and think more powerful than human beings.

\section{MACHINE LEARNING}

Machine learning is a current application of AI which promotes the reality just to be able to give machines the access to data for more ease in human work and just to learn them for themselves. Learning [2] is a key hallmark of artificial intelligence. It is an ability of the machines to take real - time data and feedback and improves performance over a time. Machine learning is type of artificial intelligence which has ability to learn and takes the data to get good output. Both the terms, Artificial intelligence and machine learning combined together very frequently when the concepts like big data, data science and analysis comes in mind. Machine learning is very efficient solution to handle such a big data in multinational industries. They actually work like a super-computer. These machines or generally known as "Humanoids" are very perfect at their work. These robots/machines can talk, answer complex questions, and multiple jobs at a time.

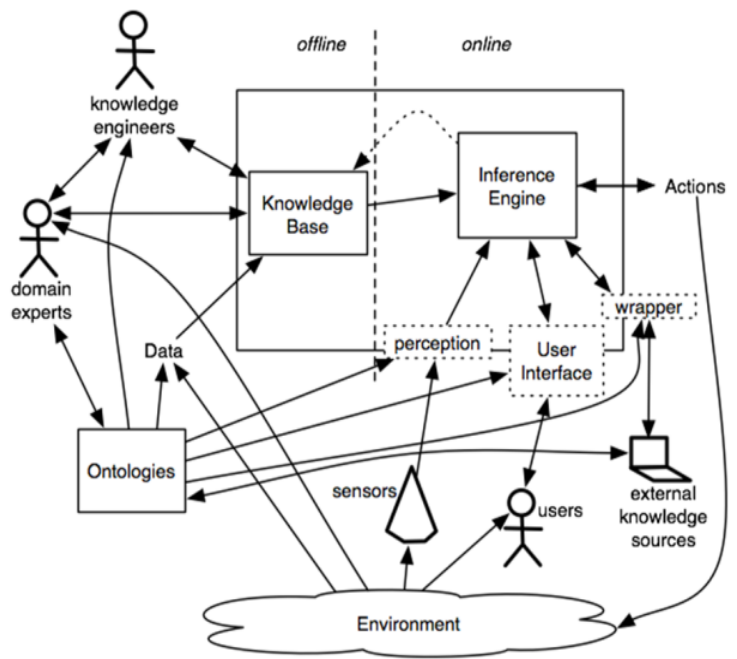

Fig.IV.a Diagram representing machine learning mechanism

The diagram explains that machine learning done not only depend on how the know ledged engineer perform on training bases but also how he works for new experiments. Machine learning is one of the most important technical approaches to AI and the basis of many recent advances and commercial applications of AI. Modern machine learning is a statistical process that helps to define the output and future use of data [3].

There are following types of learning:

1. Supervised learning.

2. Unsupervised/predictive learning.

3. Reinforcement learning.

\section{Supervised learning:}

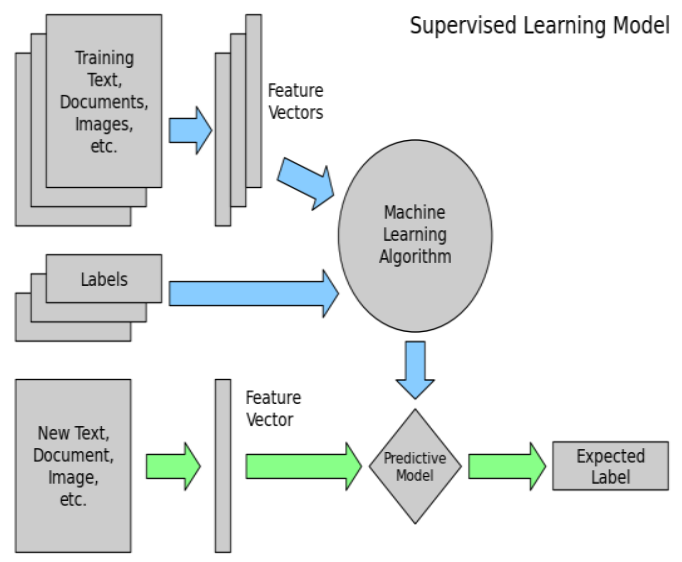

Fig. IV. b Supervised learning model 


\section{IARJSET}

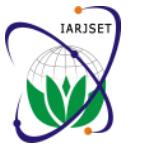

International Advanced Research Journal in Science, Engineering and Technology

National Conference on Innovative Applications and Research in Computer Science and Engineering (NCIARCSE-2017) AGTI's Dr. Daulatrao Aher College Engineering, Vidyanagar Extension, Karad

Vol. 4, Special Issue 4, January 2017

In this process, if the researchers tell the machine what the correct answer is for a particular input. It is most common technique for training neutral networks [4] and other machine learning architectures. It involves learning a mapping from a set of inputs to a target variable. The target is discrete and real value. It is solved by decision tress, naive tress [5], boosting and multi-layer [6] neutral networks.

\section{Unsupervised/predictive learning:}

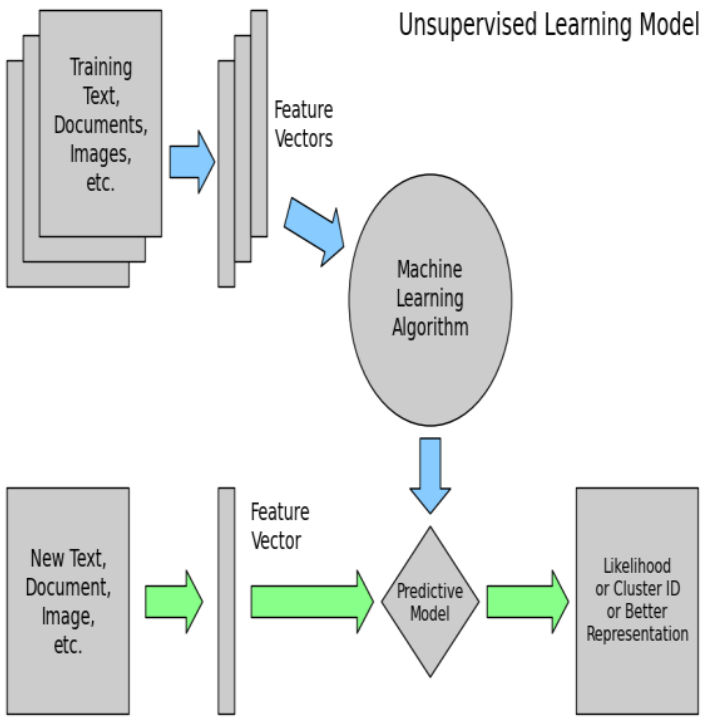

Fig.IV.c Unsupervised learning model

In this method, no labels are given to learning algorithm, leaving it on its own to find structure in its input. It can be a goal in itself i.e. hidden pattern[7] and data. Researchers don't know how to do at this moment, research is still going on. No target variables are provided. It is solved by grouping into $\mathrm{K}$ groups.

\section{Reinforcement learning:}

In this algorithm, The AI 'agent' [8] decides how to behave in order to get most of the work is done. A computer program interacts with dynamic environment in which it must perform a certain task to win against opponent. The program gives feedback in terms of punishment or reward. The machine itself selects actions to be performed for better output

\section{V.APPLICATIONS OF AI AND MACHINE LEARNING}

As we know that how $\mathrm{AI}$ is most useful topic in human life. There are many day-today examples of AI. There is siri by apple, google now by google, Watson by IBM and cortana by windows mobile for various operating systems which are intelligent digital personal assistants [9] which have speech and gesture recognition system which helps the user to find and sort out all the needed things without any physical appearance.

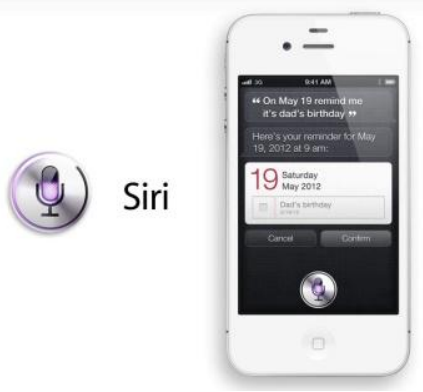

Fig.IV.a Siri by apple

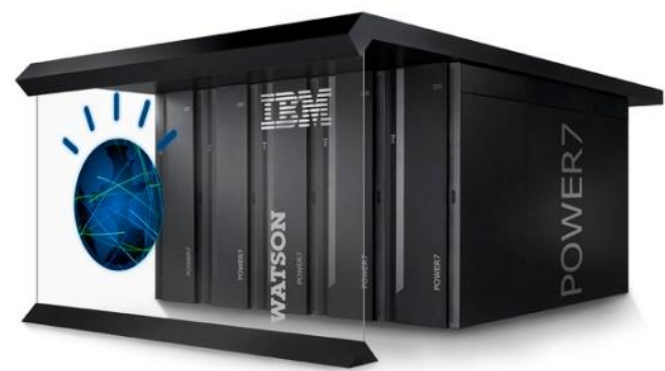

Fig, V.b Watson by IBM

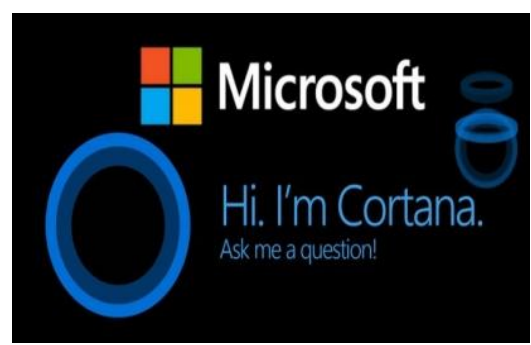

Fig.V.c Cortana by Microsoft

It will give all the information to you like...."where is nearest restaurant/college/bus station?" or it will remind your pending work, alarm clock, personal information as well as your friend's birthday or important meetings to handle, etc.

Many future and current researches are going on by scientists on humanoids i.e. robotics along with human behaviour and feelings. There are also high performance cars along with automatic drivers assists, missiles with radar, satellites and navigation systems.

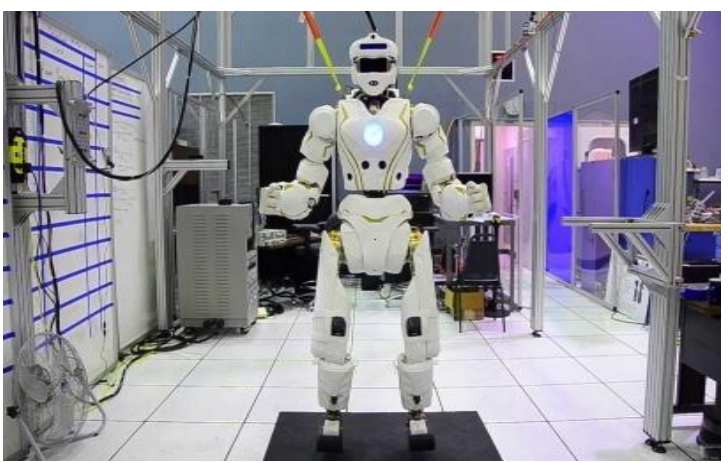

Fig.V.d. Valkyrine 


\section{IARJSET}

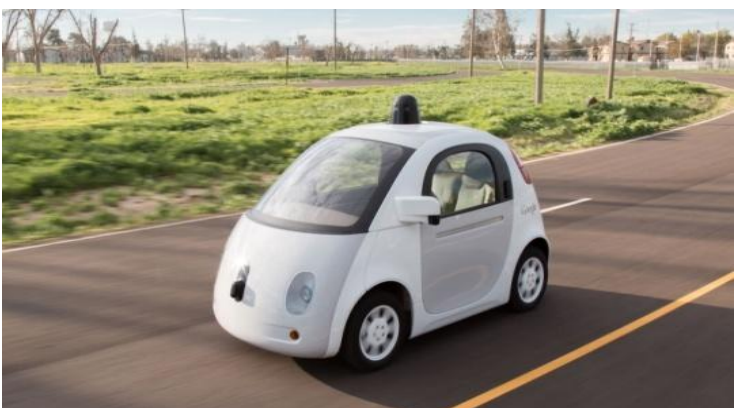

Fig.V.e Google self-driving car

The self-driving car called 'Waymo' [11] which is a google initiative drives on the road without driver. Again NASA and GOOGLE are together for first ever humanoid astronaut known as 'Valkyrine' [12] which is an absolute example of artificial intelligence.

\section{CONCLUSION}

The entire world is on the way of Digitalization and for that purpose the artificial intelligence and machine learning concepts plays an important role. Our research paper is totally based upon, how the intelligence and new machine technologies get invented in our day to day life. Today's machines are ready to give the knowledge based education and are responsible for improving the intelligence. In future, we don't think and imagine about the progress of world due to only Artificial Intelligence and Innovative Machines. We can't imagine about what happening in surrounding and in all over world because of scientists and engineers. Scientist developed the Robots who is working like a Human Being and also the research is going on to create the best world in future. Youth generation support is one of the most important parts to develop the new technologies. Combination of Science and Engineering and the quality machine learning will surely take the world at its highest fit.

\section{ACKNOWLEDGEMENT}

We are giving a sincere thanks to our Prof. Sayali S. Shinde madam and Prof Rupali N. Mahind for inspiring and sharing their wonderful thoughts regarding this subject with us. We are very thankful to our HOD of Computer Science and Engineering Dept. Resp. Prof. Ashish N. Patil Sir for helping us. A special thanks and appreciation to everyone who has contributed for this research paper.

\section{REFERENCES}

[1] Peter Norvig; Stuart Russell, "Artificial Intelligence: A Modern Approach".

[2] Sally Goldman; Yan Zhou, "Enhancing Supervised Learning with Unlabeled Data", Department of Computer Science, Washington University, St.Louis, MO 63130 USA.

[3] Niklas Lavesson,"Evaluation and Analysis of Supervised Learning Algorithms and Classifiers", Blekinge Institute of Technology Licentiate Dissertation Series No 2006:04, ISSN 1650-2140, ISBN 91-7295-083-8
[4] Bing Liu, "Supervised Learning", Department of Computer Science, University of Illinois at Chicago (UIC), 851 S. Morgan Street, Chicago

[5] T.S. Anantharman, M.S. Campbell, F.-h. Hsu, Singular extensions: Adding selectivity to brute-force searching, Artificial Intelligence 43 (1) (1990) 99-110. Also published in: ICCA J. 11 (4) (1988) 135-143.

[6] Rich Caruana; Alexandru Niculescu- Mizil,"An Empirical Comparison of Supervised Learning Algorithms", Department of Computer Science, Cornell University, Ithaca, NY 14853 USA Dissertation Series No 2006:04,ISSN 1650-2140,ISBN 91-7295083-8

[7] Zoubin Ghahramani, "Unsupervised Learning", Gatsby Computational Neuroscience Unit, University College Lond Unsupervised", "Genetic Learning Algorithms", "Reinforcement Learning and Control", Department of Computer Science, Stanford University,450 Serra Mall, CA 94305, USA.

[9] Girish Kumar jha, "Artificial Neural Networks and its applications" international journal of computer science and issues 2005.

[10] George F Ludger "Artificial Intelligence - Structures and strategies for complex problem solving" 5th Edition, Pearson, 2009.

[11] https://techcrunch.com/.../googles-self-driving-car-unit-spins-outas-way...

[12] spectrum.ieee.org/automaton/robotics/humanoids/new-r5-valkyrierobots

[13]https://www.tutorialspoint.com/artificial_intelligence/artificial_inte lligenc...

\section{BIOGRAPHIES}

Ms. Mahind Rupali N. working as assitant professor in computer science \& engineering department at Dr. Daulatrao Aher College of Engineering, karad

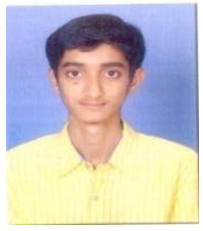

Patil Amit Umesh is currently pursuing computer science engineering at Dr. Daulatrao Aher College of Engineering, karad. The author has interest in web design and android development. 